Revue d'histoire de l'Amérique française

DEVUE D.HISTOIRE DE L'AMÉRIQUE FRANÇAISE

\title{
Les origines de la famille Le Moine (suite et fin)
}

\section{Joseph Le Ber}

Volume 1, numéro 2, septembre 1947

URI : https://id.erudit.org/iderudit/801370ar

DOI : https://doi.org/10.7202/801370ar

Aller au sommaire du numéro

Éditeur(s)

Institut d'histoire de l'Amérique française

ISSN

0035-2357 (imprimé)

1492-1383 (numérique)

Découvrir la revue

Citer cet article

Le Ber, J. (1947). Les origines de la famille Le Moine (suite et fin). Revue

d'histoire de l'Amérique française, 1(2), 257-270.

https://doi.org/10.7202/801370ar d'utilisation que vous pouvez consulter en ligne.

https://apropos.erudit.org/fr/usagers/politique-dutilisation/ 


\section{LES ORIGINES DE LA FAMILLE LE MOINE}

(suite et fin)

Depuis quelque temps les affaires d'Adrien DUCHESNE périclitaient. Déjà son père Pierre DUCHESNE avait contracté des dettes. Nous avons pu relever dans le tabellionage de BACQUEVILLE plusieurs ventes de terre entre 1613 et 1618 . Un document du 29 Avril 1617 est bien significatif à cet égard et montre à nu les embarras financiers d'Adrien DUCHESNE, c'est pourquoi nous le citons à peu près in extenso:

" Je soussigné Adrien DUCHESNE sieur d'AUZOUVILLE confesse " par la présente avoir vendu... à noble homme Maistre Jehan DELA( PORTE lieutenant particullier en la Vicomté de ROUEN... quatre " acres de terre sizes en la paroisse de SAINT OUEN en hamel d'AU( ZOUVILLE moyennant le prix de neuf vingt livres pour chacu« ne acre ...pour demeurer quitte par ledict Sieur d'AUZOU"VILLE de la somme dedeux cent soixante livres en quoy ledict " Sieur DELAPORTE seroit intervenu plégé pour moy envers le " Sieur DESPRES et pour laquelle il aurait fait arrester et constituer " prisonnier et au moien de la dicte pleige aurait esté eslargy, que " mesme pour demeurer quicte tant d'une année de la dixme montant " quarante trois livres... que de la somme de sept vingt dix livres par " moy deub aud Sieur DELAPORTE pour argent par luy à moy " presté, faisant en tout la somme de quatre cent cinquante trois livres, " et pour le surplus de la présente vendue montant à deux cent soixante " sept livres, icelle somme est demeurée entre les mains dudict Sieur "DELAPORTE. "

De plus lorsque le 31 Juillet 1642 Maître Jean DELAPORTE dât à son tour vendre le fief d'AUZOUVILLE à François de BAILLEUL c'est parce que celui-ci l'aurait saisi par décret " pour les debtes 
dudict DUCHESNE ". D'après le détail qui en est donné elles se montent à quatre mille huit cent quarante sept livres, somme importante pour l'époque.

Nous avons d'autres preuves encore de cette mauvaise situation financière: le 28 Septembre 1622 nous voyons Adrien DUCHESNE en procès avec les héritiers de son cousin Jean CASTEL; or pour décharger Maître Jean DELAPORTE lieutenant en la Vicomté de ROUEN "de plusieurs deniers qu'il aurait entièrement fraiez et déboursés depuis le commencement dudict procès jusques à la fin, montant à la somme de sept cent vingt cinq livres " il lui délaisse quatre acres et demie de terre.

Le 12 Octobre 1622, il est vrai, Jean CASTEL lui délaisse à son tour plusieurs héritages, c'est-à-dire plusieurs terres, à lui vendues par Pierre DUCHESNE le 6 Janvier 1616, à la raison de cinquante livres par acre; mais qu'on ne se fasse pas illusion, c'est Jean DELAPORTE qui avance les fonds " ayant reconnu que cessant les dits deniers" il lui eut été impossible de récupérer ce bien, comme la coutume du temps lui en laissait la faculté.

Bientôt sa mère vient personnellement à son secours, ainsi qu'on peut en juger par un acte passé le 9 Mars 1624 devant les tabellions de LONGUEUIL ${ }^{15}$; nous y lisons que "Damoiselle FOUACHE, femme et espouze de Maistre Pierre DUCHESNE Sieur d'YNBERVILLE (sic) de présent demeurante à DIEPPE donne à noble Adrien DUCHESNE Sieur d'AUZOUVILLE son fils, demeurant audit lieu, paroisse de ST OUEN PRENT EN BOURSE la somme de mille livres pour les bons et agréables services qu'elle a reçus de lui et pour ceux qu'elle espère en recevoir à l'avenir. " Cette dernière phrase est une clause de style, alors en usage pour les actes de donation.

Vers la même époque, par un autre acte dont la date n'est plus lisible elle lui " transporte en pur et loyal don " une nouvelle somme de mille livres " qu'elle a droit de prendre et avoir sur le Sieur d'IBERVILLE son mari 》.

Mais voici que par un échange avec noble homme Jean DELAPORTE, Adrien DUCHESNE lui cède le 5 Septembre $1624^{16}$ son

15. Le tabellionage de LONGUEUIL est annexé à celui de BACQUEVILLE.

16. Devant les tab. de LONGUEUIL. 
fief d'AUZOUVILLE et vingt acres de terre dont une pièce est le " chamois " du fief, dit cet acte, c'est à dire le chef mois, quelque chose comme le chef lieu, renfermant sans doute l'antique motte seigneuriale, et il reçoit en contre partie vingt cinq acres de terre avec une maison et une masure sises à AUZOUVILLE, " demeurant audit Sieur DUCHESNE, sa vie durante seullement, la quallité et nomination dudit Sieur d'AUZOUVILLE ". Autrement dit il pourra porter jusqu'à sa mort le titre de Seigneur d'AUZOUVILLE.

Il ne semble pas que cet échange ait été bien avantageux pour lui... à moins qu'il ait été fait pour compenser quelques services rendus par Jean DELAPORTE. Dans tous les cas la situation d'Adrien DUCHESNE ne se rétablit pas, les ventes de terres continuent et dès lors il n'habite plus AUZOUVILLE, sans doute depuis la mort de son père survenue avant le 18 Novembre $1626^{17}$; il réside avec sa mère au hameau de TOUS LES MESNILS, paroisse de LONGUEUIL. En $1631^{18}$ il vendit encore plusieurs acres de terre; en mars 1634 un nommé BARJAMIN du THIL, reconnaissait lui devoir 15 sous de rente; puis il semble avoir disparu de la région; le tabellionage de BACQUEVILLE n'enregistre plus aucun acte de lui. C'est donc après cette date sans doute qu'il laissa la France, probablement cette année là, car dans sa "Relation de la Nouvelle France " pour 1634, le Père LEJEUNE mentionne sa présence à QUEBEC. Il se serait embarqué en Avril ou Mai sur la flotte des Cent Associés pour aller au Canada refaire sa fortune ou tout au moins une existence digne. Heureuses dettes, heureuse ruine, pourrait-on dire; en partant pour le Canada où il allait devenir chirurgien de la colonie (avait-il une compétence spéciale en cet art?) il y attirerait bientôt son neveu Charles LE MOINE fondateur d'une des plus illustres familles canadiennes. DIEU sait toujours tirer le bien du mal.

Avant d'en finir avec Adrien DUCHESNE nous voulons dire quelques mots encore sur la date de son départ de FRANCE et celle de sa mort: FAILLON ${ }^{19}$ signale que le 9 Février 1631 « la femme du

17. A cettè date un acte du T.B. le mentionne comme décédé, et cette année-là Adrien DUCHESNE rendit aveu à la Seigneurie d'OMONVILLE, sans doute comme l'héritier de son père.

18. T.B. 22 Septembre 1631.

19. Histoire de la Colonie Française en Canada (VILLEMARIE 1865) T.I. p. 252 . 
chirurgien anglais Adrien DUCHESNE " fut marraine d'Elisabeth COUILLARD, mais il ne donne pas le texte de l'acte de baptême. Cette affirmation nous étonne d'abord parce qu'Adrien DUCHESNE n'était pas Anglais et ensuite parce qu'un acte du tabellionage de BACQUEVILLE nous prouve que le 22 Septembre 1631 il était encore en France. Serait-il donc arrivé au Canada avant la prise de QUEBEC ? Comment concilier sa présence au Canada en Février 1631 et en France en Septembre de la même année? Comment serait-il revenu de là-bas alors que QUEBEC était occupé par les Anglais à cette époque? Et quelle était cette femme d'Adrien DUCHESNE? L'aurait-il emmenée au Canada? S'était-il remarié? L'aurait-il laissée au Canada en supposant qu'il y soit venu avant la prise de QUEBEC et en soit reparti en même temps que CHAMPLAIN? Autant de questions auxquelles nous ne pouvons répondre; une chose est certaine pour nous c'est que le 22 Septembre 1631 il était à LONGUEUIL.

Quelle est la date de sa mort? On sait qu'en 1641 il appela au Canada son neveu Charles LE MOINE. C'est probablement cette année-là ou la suivante qu'il mourut; là encore le tabellionage de BACQUEVILLE nous apporte une indication; à la date du 14 Avril 1642 nous y trouvons un acte commençant ainsi: " Fut présent Maistre Jehan DUCHESNE demeurant en la ville de DIEPPE soy disant hérittier par bénéfice d'inventaire de feu Adrien DUCHESNE vivant sieur d'AUZOUVILLE ". Et ce texte confirme encore ce que nous disions de la fortune d'Adrien DUCHESNE: elle était fort obérée puisque son fils ne crut devoir accepter la succession que sous bénéfice d'inventaire... cependant il en subsistait quelques lambeaux puisque ce fils Jean DUCHESNE rendait par la suite aveu pour des terres qui avaient appartenu à son père.

Mais à qui était passé le titre de Seigneur d'IBERVILLE porté par la père d'Adrien DUCHESNE? C'est ce que nous allons voir maintenant.

PIERRE II DUCHESNE : Il y aurait alors d'autres DUCHESNE à DIEPPE, issus sans doute de la même souche. L'un deux, Pierre (que nous appellerons PIERRE II) a laissé quelques traces dans les actes notariés et ceux de l'Etat civil de DIEPPE. Le chroniqueur ASSELINE le mentionne à plusieurs reprises car il fut échevin et procureur syndic de la ville de DIEPPE. Etait-il parent d'Adrien DUCHESNE? C'est fort probable. En tout cas il ne peut être con- 
fondu avec le père d'Adrien DUCHESNE qui portait aussi le prénom de Pierre. Nous avons plusieurs actes qui le prouvent et de plus il vécut jusqu'en 1665 alors que le premier Pierre mourut en 1626.

Dès 1622 Pierre DUCHESNE avocat et procureur syndic des habitants de la ville de Dieppe rendait aveu 20 aux chanoines de SAUQUEVILLE. Ce titre de procureur syndic de Dieppe qu'il continua à porter nous permet de ne pas le confondre avec le père d'Adrien.

Voici de plus un accord conclu devant les tabellions de Dieppe le 31 Août $1665^{2}$, après la mort du second Pierre DUCHESNE, entre " Maistre François DUCHESNE, sieur d'HIBERVILLE, Conseiller assesseur au bailliage de Caux, vicomté d'Arques, fils ainé et héritier de Maistre Pierre DUCHESNE ancien conseiller eschevin de la ville de DIEPPE, et honneste femme Catherine CANU veuve de feu Maistre Jacques DUCHESNE advocat, fils puisné dudit feu Sieur DUCHESNE, père. " Un autre acte fut passé le 3 Octobre 1665 devant les mêmes notaires pour constituer " les lots et partages des maisons, masures, héritages et rentes assis aux paroisses de SAUQUEVILLE, TOURVILLE, OFFRANVILLE, ST MARDS, AUFFAY, PELLETOT et LONGUEIL, provenants de la succession de défunt Maistre Pierre DUCHESNE en son vivant sieur d'YBERVILLE et SAUQUEVILLETTE, et ancien Conseiller eschevin de ville de DIEPPE.) C'est ce partage qui nous apprit que le fief d'IBERVILLE appartenant aux DUCHESNE se trouvait sur la paroisse de TOURVILLE. Comment était-il passé à cet autre Pierre DUCHESNE? Si le tabellionage de Dieppe existait encore pour la première moitié du XVIIe siècle, nous le saurions probablement, car il est à croire que Pierre Ier le vendit à son parent Pierre II à l'époque ou les affaires d'Adrien allaient mal.

Enfin voici encore un acte tiré du tabellionage de Dieppe qui dénote une proche parenté entre Maistre PIERRE II DUCHESNE ancien conseiller échevin et Judith DUCHESNE sœur d'ADRIEN. Nous le donnons ici entièrement parce qu'il est fort intéressant à d'autres points de vue:

20. Archives de la Seine-Inférieure G. 9398.

21. Les registres des tabellionages de Dieppe et d'Arques n'existent plus et avec beaucoup de lacunes - qu'a partir de 1654, sans quoi nous aurions pu y faire une ample moisson de renseignements. 
RAQUIS D'UNE RENTE DE TRENTE LIVRES PASSE DEVANT LES TABELLIONS DE DIEPPE

\section{LE 13 MAI 1664}

Fut Présent Jean ASSEGOND Me chirurgien demeurant au POLLET paroisse de NEUFVILLE pr spécialement fondé de Jacques et Charles le moine frères, Jacques LEBER ayant espouzé Jeanne le moine et Anne Le Moine ayant épousé Michel Messier détenu de présent captif par les sauvages hyroquois de la Nouvelle France, lesdita Jacques et Charles le MOINE, Jeanne et Anne leMOINE enfans de défunt Pierre le MOINE et cohéritiers en la succession de deffunte Judith DUCHESNE leur mère demeurant et résidant de présent en ladite Nouvelle France en l'isle de MONTREAL, par procuration passée par devant Benigne BASSET commis au greffe du tabellionage de VILLEMARIE pour Messieurs de la Cie de ladite Nouvelle France en ladite isle de MONTREAL le quatriesme jour de septembre mil six cens soixante et deux de laquelle procuration il a promis bailler coppie coll. à ses dépens au Sieur DUCHESNE cy après nommé:; voll. a recongnu et confessé avoir reçue présentement comptant de Maistre Pierre DUCHESNE antien Conseiller eschevin de ceste ville de DIEPPE aussy présent ce stipullant, c'est assavoir la somme de cent cinquante livres tournois pour le raquis et amortissement du principal de dix livres quatorze sols trois deniers de rente hypotecque au denier quatorze, faisant partie de trente livres de rente au dernier quatorze, en quoy ledit Sieur DUCHESNE s'est constitué vers lesdits deffunts LEMOINE et Judith DUCHESNE sa femme pour le dot et rapport de mariage d'icelle DUCHESNE en contractant leur mariage suivant et pour les causes contenues et mentionnées en leur traicté soubs seing privé le dixième d'octobre mil six cent dix huit recongnu devant le ROUX et VAULTIER lors tab. à DIEPPE le vingt quatriesme de Janvier mil six cens dix neuf, insignuer aux assises du baillage de Caux Vicomté d'Arques le quatriesme jour de Mars aud. mil six cens dix neuf, de laquelle somme de cent cinquante livres ledit ASSEGOND en ladite qualité s'est tenu a content et bien payé, en a tenu quitte et d'aultant dechargé le dit Sieur DUCHESNE et tous autres au moyen de quoy a consenti et accordé que le traité de mariage des dicts deffuncts LEMOINE et Judith DUCHESNE par luy porté, ensemble celuy porté par ledit 
Sieur DUCHESNE, minutte d'iceluy et tous autres pour faisant mention desdicts trente livres de rente de dot esmargés en sa présence ou absence constituant à ceste fin pour procureur auxdits Lemoine Frères et sœurs le porteur du présent parce que lesdits adménagements à la présente quittance ne vaudront que pour un seul et mesme acquis se submettant iceluy ASSEGOND faire ratiffier et avoir agréable ces présentes toutesfois et quantes ausdits LE MOINE Frères et sœurs à en fournir acte et ratiffication audit Sieur DUCHESNE pour luy servir de plus grande assurance et garantie dudit présent raquis. Et en présence et du consentement de Maistre Jean BELLIARD procureur commis aux jurisdictions Royalles d'Arques cy devant receveur des consignations de la Vicomté d'Arques, entre les mains duquel la somme de quatre cens vingt livres pour le raquis desdits trente livres aurait esté garni suivant la sentence qu'il a dict avoir esté donnée au Siège du baillage de DIEPPE le jour de mil six cens..."

En marge il y a un reçu donné par ASSEGOND le 19 Juillet 1664 de 186 Livres pour le raquis de 13 Livres 5 Sols 9 deniers faisant partie de 30 Livres.

Si Pierre II DUCHESNE avait constitué cette rente de 30 Livres pour la dot de Judith DUCHESNE en 1618, c'est que déjà le père de celle-ci ne se trouvait pas très argenté. ou bien parce Pierre II s'était trouvé chargé de cette rente en achetant IBERVILLE ou une autre terre. Pourtant l'acte ci-dessus ne le dit pas.

Nous avons rencontré de 1627 à 1637 dans les actes de baptême de la paroisse ST REMI un Pierre DUCHESNE avocat, époux de Marie MONTFORT, mais ce ne peut être notre Pierre II, car le rédacteur de ces actes n'eut pas manqué de lui donner ses titres soit de Conseiller échevin soit de procureur syndic de la ville de Dieppe; de plus aucun de ses quatre enfants baptisés alors n'est mentionné dans les actes de succession avec François et Jacques DUCHESNE Fils de Pierre II mais cependant comme Marie MONTFORT fut marraine de Charles LE MOINE le 2 Août 1626 c'est une preuve supplémentaire qu'il y avait quelque parenté entre ce Pierre DUCHESNE et Judith DUCHESNE.

De Judith DUCHESNE sœur d'Adrien et mère de Charles LE MOINE nous ne savons rien, si ce n'est le peu que nous dirons de son mariage avec Pierre LE MOINE, mais elle fut la mère d'au moins 
sept enfants dont Charles LE MOINE et c'est là un beau titre de gloire.

\section{LA FAMILLE LE MOINE}

Nous savons fort peu de choses sur la famille de Pierre LE MOINE père de Charles.

Les LE MOINE qui écrivaient leur nom indifféremment LEMOINE, LE MOINE ou LE MOYNE (on trouve parfois ces trois orthographes dans le même acte) étaịent très nombreux à DIEPPE aux XVIè et XVIIè siècles; il s'en trouvait dans la noblesse, dans la bourgeoisie et dans le peuple. Peut-être avaient-ils un ancêtre commun, mais il ne nous a pas été possible de le prouver; nous savons seulement que la branche anoblie au milieu du XVIè siècle était originaire d'ANNEVILLE/SCIE ${ }^{2}$; si elle avait quelque parenté avec les autres branches, il faudrait remonter jusqu'au XVè siècle pour la découvrir, nous le savons d'autant mieux que nous avons pu établir sa généalogie depuis cette époque. Aucun document ne nous a permis de relier les unes aux autres avec certitude les différentes branches. Or à l'époque où vivaient Pierre LE MOINE et sa femme il y avait à DIEPPE une bonne vingtaine de foyers portant ce nom patronymi$q^{2 u e}{ }^{23}$. Nous pouvons tout au plus déceler une parenté asssez vague entre certains d'entre eux sans pouvoir en préciser le degré.

L'acte de remboursement de rente transcrit ci-dessus et portant la date du 13 Mai 1664 nous apprend que le contrat de mariage entre Pierre LE MOINE et Judith DUCHESNE avait été conclu le 10 Octobre 1618 et reconnu devant les tabellions de DIEPPE le 24 Janvier 1619 , on peut donc affirmer qu'ils s'étaient mariés au début de 1619 , malheureusement les registres du tabellionage de DIEPPE pour cette époque ont disparu, sans quoi nous aurions connu saps doute la date exacte de ce mariage et beaucoup d'autres détails intéressants sur leur famille et leur situation; et comme cet acte le prouve, en 1664 ils étaient décédés tous les deux.

22. Canton de LONGUEVILLE / SCIE à $10 \mathrm{Kms}$ environ de DIEPPE.

23. Les notes présentes ont été recueillies avant 1940; peut-être aurions-nous pu trouver quelques éclaircissements dans certains arrêts du Parlement de Normandie, mais pendant toute la durée de la guerre il a été impossible de les consulter et au moment où nous écrivons ces lignes ils ne sont pas encore accessibles. 
A notre connaissance ils eurent au moins sept enfants, ce $\varepsilon \subset \mathrm{Cr}$. Io) François, baptisé en l'Église ST REMI de DIEPPE le 23 Février 1621. Il eut pour parrain Maitre Jean PETIT et pour marraine Catherine FLEURYE.

2o) Jacques dont l'acte du 13 Mai 1664 nous apprend l'existence; il y est nommé avant Charles. Il peut y avoir une lacune, chose fréquente, dans les registres de baptême. Il se peut aussi qu'il ne soit pas né à DIEPPE.

3o) NICOLAS baptisé à St REMI le 9 Août 1624. Son parrain fut Nicolas ALLAIS et sa marraine Geneviève DABLON.

4o) Charles, baptisé à St REMI le 2 Août 1626. Il fut nommé par Charles LEDOUX et Marie MONTFORT.

5o) Jeanne, baptisée à ST REMI le 24 Avril 1630; elle eut pour parrain VIDAL LE PREVOST et pour marraine Jeanne LECARPENTIER.

6o) Anne, baptisée à ST REMI le 17 Novembre 1632 nommée par Jean LE MOYNE et Anne THEROULDE.

7o) Léonard, baptisé à ST JACQUES DE DIEPPE le 6 Janvier 1642. Son parrain fut Maître Léonard FAVIER, Contrôleur. Il mourut onze jours plus tard et fut inhumé dans l'Église.

Parmi les noms de tous les parrains et marraines de ces enfants, trois seulement nous sont un peu connus:

Geneviève DABLON, parce qu'un membre de sa famille Claude DABLON fut syndic de DIEPPE, un autre missionnaire au Canada et d'autres encore eurent quelque notoriété.

Marie MONTFORT était la femme de Pierre DUCHESNE avocat, que nous n'avons pas cru pouvoir identifier avec Pierre II DUCHESNE, Sieur d'IBERVILLE; mais sa présence au baptême de Charles LE MOINE est une présomption de parenté.

Enfin un Jean LE MOYNE est parrain d'Anne, mais plusieurs LEMOINE à DIEPPE portaient ce prénom de Jean. Il nous est donc impossible de préciser à quel foyer il appartenait.

On observera aussi qu'un espace de presque dix années s'étend entre la naissance d'Anne et celle de Léonard ce qui n'a rien d'extraordinaire mais laisse supposer qu'il a pu y avoir d'autres enfants entre eux. De plus ce Léonard fut baptisé en l'Église St Jacques, c'est donc que ses parents avaient changé de paroisse. A quelle date exactement? FAILLON dit que ce fut en 1633 mais à notre connaissance rien 
ne l'indique dans les registres de ST JACQUES. Que s'était-il passé? Un revers de fortune? Est-ce à cause de cela que l'année précédente Charles s'était embarqué pour le Canada? Léonard fut inhumé dans l'Eglise, par conséquent ses parents tenaient encore un rang social assez élevé.

C'est tout ce que nous savons de précis sur le foyer de Pierre LE MOINE et ce peu est sans doute déjà connu. Néanmoins en cherchant à identifier leur hôtellerie nous arriverons peut-être à percer un peu l'obscurité qui plane sur leur vie et leur famille.

\section{L'HÔTELLERIE DES LE MOINE}

On a souvent dit que le père de Charles LE MOINE étaịt hôtelier ou cabaretier; on laisse parfois entendre qu'il servait à boire aux matelots du port dans quelque taverne de troisième ou quatrième ordre. Tâchons de savoir ce qui en était.

Souvent nous nous sommes demandé quelle était cette hôtellerie; quelle était son enseigne, et où elle se trouvait. Le hasard nous mit un jour sur sa piste; dans une liasse de l'Amirauté du Havre, aux archives de la Seine-Inférieure, concernant un procès fait aux Espagnols pour une expédition au Chateau de la Mine (Guinée) en 1583, se trouve une enquête faite par un membre du Parlement de Normandie, Maximilien de Limoges ${ }^{24}$. On y lit ceci: "...le samedi 19 Octobre 1585 nous nous sommes acheminés vers ladite ville de Dieppe et avons pris notre logis en la maison ou pend pour enseigne l'Image Sainct Elloy et dont est maistre Jehan le Moine ". C'est là que Maximilien de Limoges convoqua les témoins et nota les dépositions. Ce texte nous apprend donc que vers la fin du XVI ${ }^{\circ}$ Siècle un LE MOINE tenait à DIEPPE une hôtellerie où ne dédaignaient pas de descendre de hauts magistrats et cette hôtellerie avait comme enseigne l'Image St Eloi; il se peut que ce Jean LE MOINE soit le père ou le grand père de notre Pierre LE MOINE. Cependant nous ne pouvons affirmer encore que l'hôtellerie en question soit précisément celle où naquit et grandit Charles LE MOINE de LONGUEUIL, pas plus que nous ne pouvons dire si le Maistre de ce logis est le Jean LE

24. Maximilien de Limoges avait épousé une Damoiselle LE MOINE de la branche noble. 
MOYNE qui, d'après les registres de St Remi épousa le 7 Octobre 1576 Guillemette HENAULT.

Pourtant Pierre LE MOINE fut bien le maître de l'hôtellerie portant pour enseigne l'Image St Eloi; le tabellionage de DIEPPE nous en fournit plusieurs preuves. Ainsi au 4 Janvier 1656 se trouve un acte ainsi conçu:

" Laurent d'Estrépagny escuyer, Sieur de la Gravière estant de présent en cette ville de Dieppe logé au logis ou pend pour enseigne l'Image Sainct Esloy au devant du Puis sallé de la dite ville de Dieppe, noble homme François DANIEL capitaine pour le Roy en la marine; honorable homme Pierre LE MOYNE du logis l'Image Saint Esloy et Pierre PERIER bourgeois demeurant au dict Dieppe " donnent une attestation au Sieur Pierre DEBILA marchand demeurant à ROUEN de présent audit DIEPPE; logé audit logis l'Image Saint Esloy... ) etc.

L'hôtellerie de Pierre LE MOINE est donc bien celle qui a pour enseigne l'Image St Eloi, et par surcroît nous savons maintenant qu'elle était située sur la Place du Puits Salé: peut-être arriveronsnous à connaitre son emplacement exact.

Nous avons vu qu'au XVI ${ }^{\circ}$ Siècle cet hôtel était assez bien coté puisqu'un membre du Parlement de Normandie pouvait y descendre. Lorsque Pierre LE MOINE le dirigeait sa clientèle n'était pas moins distinguée, l'acte ci-dessus nous montre que pouvaient s'y rencontrer des gentilhommes, des capitaines de navire et des marchands. Nous en avons recueilli plusieurs autres aussi probants. C'est ainsi que le 11 Septembre 1662 plusieurs hôtes de marque viennent y règler leurs affaires: c'est Isaac de FURET Ecuyer, Sieur de BAUDRIBOSC demeurant à ST PIERRE LE VIGER; ${ }^{25}$ ce sont Messire Charles de BRETIGNIERES, Chevalier Seigneur de la Pertuisane et Dame Marie de BERRUYER son épouse, la propre fille de feu Messire Jacques de BERRUYER en son vivant écuyer, seigneur de Manselmont Conseiller du roi Louis XIII en tous ses Conseils, l'un des Directeurs des grandes Sociétés Commerciales fondées par RICHELIEU, Compagnie des Iles de l'Amérique, Compagnie des CentAssociés etc...

25. Canton de FONTAINE LE DUN. 
Le 27 Août 1663 c'est François LESTIBOUDOIS Sieur de la Vallée, l'un des principaux colons de la Martinique où il est capitaine d'une compagnie, qui règle une affaire de pétun avec Jacques et Jean MEL, gros marchands de DIEPPE.

Enfin le 18 Novembre 1667 nous y trouvons Messire Charles de BIRAN de Casteljaloux, Chevalier, Capitaine d'une compagnie de Chevaux-léger.

Une hirondelle ne fait pas le printemps, dit-on, mais les exemples que nous apportons ici ne sont-ils paș suffisants pour démontrer que l'hostellerie de Pierre LE MOINE non seulement n'était pas un vulgaire cabaret, mais qu'elle était fréquentée par la bonne Société de l'Epoque et que si en son enfance Charles LE MOINE de LONGUEUIL $\mathrm{y}$ entendit raconter maints voyages au long cours, maintes aventures, dans les pays lointains, ce fut probablement de la bouche même des fondateurs de nos plus anciennes colonies et c'est peut être chez son beau frère qu'Adrien DUCHESNE fit connaissance de l'illustre CHAMPLAIN et des Pères Jésuites qui évangélisaient le CANADA.

Il nous reste maintenant à repérer l'adresse exacte de cette hôtellerie. Un contrat de vente trouvé chez GOSSELIN bouquiniste rue Ganterie à ROUEN, dans un ballot de vieux titres de propriété va nous y aider grandement. Cet acte que nous avons cru utile de transcrire entièrement, nous le donnons en appendice; ${ }^{25 \mathrm{a}}$ qu'il nous suffise d'en extraire ici les détails les plus intéressants.

Le 19 Novembre 1681 les enfants de Pierre LE MOINE Capitaine pour le Roi en la marine vendent à Nicolas BOURDIN Marchand bourgeois de DIEPPE, pour le prix de 8.500 Livres la maison et hotellerie ou pend pour enseigne l'Image St Eloi sise à DIEPPE, grande rue, paroisse St Remi, bornée par le Sieur CHAUVIN, le Sieur de la Houssaye et l'hotellerie du Chariot d'Or. Cette hotellerie a été vendue à BARTHELEMY LE MOINE, ayeul des vendeurs par noble homme Jean CHAUVIN Conseiller Secrétaire du Roi le 2 Novembre 1611. Actuellement y est-il spécifié elle est " en totale décadence » et a grandement besoin de réparations.

Parmi les charges dont elle est grevée figurent dix livres de rentes dues à l'hôtel commun de la ville de Dieppe pour le droit de la fontaine

25a- La Revue publiera, dans sa prochaine livraison, sous la rubrique des ‘Documents inédits», ce Contrat de vente. 
qui est dans la maison. Ce détail est précieux; nous savions déjà qu'elle se trouvait devant le Puits Salé et nous avons vu quels étaient ses abornements, or si nous nous reportons maintenant au "Livre des Fontaines, de la ville de Dieppe qui se trouve à la Bibliothèque Municipale, nous y trouvons un mémoire sur l'Etat des tuyaux daté du 20 Mai 1652 et nous y lisons ceci: "No 13 - L'adjus de la fontaine de la Maison nommée ST ELOY apartenant à Monsieur le Capitaine LE MOYNE est au droit de la grande porte ) (Cette maison est bornée par celles de $\mathrm{M}$. de la HOUSSAYE, de M. le Conseiller MARTIN et de M. le Lieutenant DABLON). Nous pensons que malgré l'incendie de 1694 la topographie de l'endroit a peu changé; l'une des maisons porte encore des armoiries, nous pensons que ce sont celles de M. de la HOUSSAYE. Chose curieuse, de l'autre côté de la rue, un pharmacien a pris comme enseigne l'Image de ST ELOI; ce qui indique bien une tradition.

Le contrat de vente nous apprend en outre que BARTHELEMY LE MOINE acheta cette hôtellerie le 2 Novembre 1611 à noble homme Jean CHAUVIN et que à sa mort elle passa à son fils Pierre LE MOINE ${ }^{26}$ qu'on ne peut confondre avec Pierre LE MOINE

26. Voici quelques notes sur Pierre LE MOINE Capitaine pour le Roi en la Marine. Elles peuvent être intéressantes dans cette revue puisque lui aussi alla plusieurs fois au CANADA.

Nous n'avons pas trouvé son acte de baptême mais ceux de ses frères et sœurs au nombre de six. Son père fut Barthélémy LE MOINE Marchand de DIEPPE et sa mère Catherine DELAMARE. Sa naissance doit être placée avant 1614 ou peut-être entre 1614 et 1616 donc en 1615. Il épousa Marie MARTIN le 23 février 1637 à St-Rémy de DIEPPE.

Dans les années 1644-1645-1646 (Tabellionage de DIEPPE 20 Septembre 1661) il va au Canada avec le navire " Le Marinier " qu'il commande. Ce navire étant sur son départ pour aller à CORFOU se perdit en la rade du Havre. Selon un article de la revue "La Normandie " (Janvier 1896: le Chevalier de Grémonville, par Legrelle p. 16 notes), il fit quand même le voyage ayant pris le commandement d'un autre navire.

Nous le retrouvons au Canada en 1664. Un a Etat des vaisseaux du port du Havre daté de 1664 porte cette mention " Le navire le St Jean Baptiste de 300 tonneaux et 24 pièces de canon, capitaine Pierre LE MOIGNE de DIEPPE... de présent en voiage au Canada " (cf Sté Histoire de Normandie, Mélanges (1907) pages 28 et 29 ).

Ceci nous est confirmé par un article de l'Abbé Auguste GOSSELIN (cf revue catholique de Normandie 1892 p. 261, les Normands au Canada) dans la biographie de Jean BOURDON, colon au Canada; celui-ci s'embarqua à QUEBEC cette annéelà 1664 sur le navire nommé le ST JEAN BAPTISTE où commandait le capitaine LEMOIGNE de DIEPPE (LEMOIGNE est une déformation de LEMOINE, encore courante en Normandie). Il est certain que ses voyages furent nombreux la et ailleurs. Le 24 Mars 1663 (T.D.) Pierre LE MOINE Capitaine d'un navire 
Maitre de l'Hôtellerie, puisqu'il était Capitaine en la marine. Est-ce qu'il y aurait un lien de parenté entre eux? C'est probable mais aucun document ne nous permet de l'affirmer comme aucun non plus ne nous apprend à quelle date ce dernier est venu l'habiter. Malheureusement, car nous ne pouvons par suite, assurer que Charles LE MOINE d€ LONGUEUIL est bien né à cet endroit, quoique la chose soit assez probable.

D'autres plus heureux que nous découvriront peut-être un jour des indications plus précises à ce sujet. Quant à nous, fort heureux d'avoir pu sauver ces modestes notes après les bombardements de notre presbytère en 1940 et de notre refuge en 1944, nous préférons les publier incomplètes plutôt que de les perdre pensant être utile ainsi aux futurs chercheurs.

Evidemment dans cette étude plusieurs points restent encore obscurs: la date de départ d'Adrien DUCHESNE pour le Canada, le degré de parenté entre Jean LE MOINE le premier Maitre de l'hotellerie ainsi que la date exacte où celui-ci commença à l'habiter. En tout cas ces notes ont apporté quelque lumière sur la question des origines de Charles LE MOINE de LONGUEUIL, en particulier, et elles nous ont fait comprendre que s'il porta au Canada les Noms de LONGUEUIL et d'IBERVILLE c'est en souvenir de sa famille maternelle qui parait ainsi lui avoir été plus chère que celle de son père, peutêtre parce qu'elle occupa un rang social plus élevé.

Abbé Joseph LE BER

Curé de Veules-les-Roses (Seine-Infre)

de 200 Tonneaux nommé le St Louis de présent au port de Dieppe, signe avec les propriétaires et fournisseurs de ce navire une charte partie " pour aller à droite route au SENEGAL et Capde Vert ".

Il y retourne en 1668 et 1669 (cf T.D. 171311668-2.I.1669) sur le navire də FLEURISSANT. Le Voyage de 1669 fut le dernier: " parti de DIEPPE le 16 Mars 1669 pour le Sénégal, il y arriva le $30 \mathrm{Mai}$ y déchargea son navire le rechargea de cuivre et de morfil (ivoire), mais il tomba malade et décéda le 30 Aout. Avant de mourir il confia le commandement de son navire au Maitre des Matelots, Antoine DANDASNE, lequel à son retour fit son rapport à l'Amirauté du Havre le 21 Novembre 1669 " (cf GOSSELIN Notes sur la Marine Normandie p. 156). 